\title{
Approximate analytical solution of the fractional epidemic model
}

\author{
S. Z. Rida ${ }^{1}$, A.S. Abdel Rady ${ }^{1}$ A.A.M. Arafa ${ }^{*}$, and M. Khalil ${ }^{2}$ \\ ${ }^{1}$ Department of mathematics, Faculty of Science, \\ South Valley University, Qena, Egypt \\ Email: szagloul@yahoo.com, anaszi2@yahoo.com \\ ${ }^{2}$ Department of mathematics, Faculty of Engineering, \\ Modern Science and Arts University (MSA), Giza, Egypt \\ m_kh1512@yahoo.com
}

\begin{abstract}
In this paper an analytical expression for the solution of the fractional order epidemic model of a non-fatal disease in a population which is assumed to have a constant size over the period of the epidemic is presented. Homotopy analysis method (HAM) is implemented to give approximate and analytical solutions of the presented problem.
\end{abstract}

Keywords: Homotopy analysis method, Fractional order ordinary differential equations, Models of infectious diseases.

\section{Introduction}

Mathematical models of infectious diseases [2] can provide important insight into our understanding of epidemiological processes, the course of infection within a host, the transmission dynamics in a host population, and formulation or implementation of infection control programs. The model presented here can be used to model any infectious disease of humans or wildlife with discrete disease states, irrespective of the number of disease states. The problem of spreading of a non-fatal disease in a population which is assumed to have constant size over the period of the epidemic is considered in [4]. At time $t$ suppose the population consist of:

$x(t)$ which is the number of susceptible individuals; 
$y(t)$ which is the number of infected individuals at time " $t$ " and are able to spread the disease by contact with susceptible;

$z(t)$ which is the number of isolated individuals, who cannot get the disease or transmit it either they have a natural immunity, or they have recovered from the disease and are immune from getting it again, or they have been placed in isolation, or they have died. The mathematical model does not distinguish between these possibilities.

Assume there is a steady constant rate between susceptible population and infective population and that a constant proportion of these constant result in transmission. Then in time $\delta \mathrm{t}, \delta \mathrm{x}$ of the susceptible population become infective, where

$$
\delta x=-\beta x y \delta t
$$

If $\beta>0$ is the rate of infection and $\gamma>0$ is the rate at which current infective population become isolated, then:

$$
\delta y=\beta x y \delta t-\gamma y \delta t
$$

The number of new isolated population, $\delta z$ is given by:

$$
\delta z=\gamma y \delta t
$$

Now let $\delta t \rightarrow 0$, then the following nonlinear system of ODEs determines the progress of the disease:

$$
\begin{aligned}
& \frac{d x}{d t}=-\beta x y, \\
& \frac{d y}{d t}=\beta x y-\gamma y, \\
& \frac{d z}{d t}=\gamma y .
\end{aligned}
$$

Subject to

$$
x(0)=N_{1}, y(0)=N_{2}, z(0)=N_{3} .
$$

Biazar [1] used the Adomian decomposition method (ADM) to solve (1) while the authors in [7] applied the variational iteration method (VIM). In [8] the homotopy perturbation method (HPM) was employed to get an approximate solution of (1).

The rest of the paper is organized as follows. In Section 2, a discussion about the fractional calculus theory is presented. The idea of homotopy analysis method (HAM) for solving a system of fractional order ordinary differential equations is 
discussed in section 3. In Section 4, we introduce fractional-order into the model of non-fatal epidemic and the approximate solution of the model is presented. Section 5 is devoted for the numerical results and discussion.

\section{Fractional calculus}

Differential equations of fractional order have been the focus of many studies due to their frequent appearance in various applications in fluid mechanics, viscoelasticity, biology, physics epidemiology [2] and engineering. Consequently, considerable attention has been given to the solutions of fractional ordinary differential equations, integral equations and fractional partial differential equations of physical interest. Recently, a large amount of literatures developed concerning the application of fractional differential equations in nonlinear dynamics. The differential equations with fractional order [5] have recently proved to be valuable tools to the modeling of many physical phenomena. This is because of the fact that the realistic modeling of a physical phenomenon does not depend only on the instant time, but also on the history of the previous time which can also be successfully achieved by using fractional calculus. Most fractional differential equations do not have exact analytic solutions, so approximation and numerical techniques must be used. The variational iteration method (VIM), the Adomian decomposition method (ADM) and Homotopy analysis method (HAM) [6] are relatively new approaches to provide an analytical approximation to linear and nonlinear problems, and they are particularly valuable as tools for scientists and applied mathematicians, because they provide immediate and visible symbolic terms of analytic solutions, as well as numerical approximate solutions to both linear and nonlinear differential equations without linearization or discretization. There are several definitions of a fractional derivative of order $\alpha>0$. The two most commonly used definitions are Riemann-Liouville and Caputo. Each definition uses Riemann-Liouville fractional integration and derivatives of whole order. The difference between the two definitions is in the order of evaluation. Riemann-Liouville fractional integration of order $\alpha$ is defined as:

$$
\begin{aligned}
& J^{\alpha} f(x)=\frac{1}{\Gamma(\alpha)} \int_{0}^{x}(x-t)^{\alpha-1} f(t) d t, \quad \alpha>0, x>0, \\
& J^{0} f(x)=f(x) .
\end{aligned}
$$

The next two equations define Riemann-Liouville and Caputo fractional derivatives of order $\alpha$, respectively,

$$
D^{\alpha} f(x)=D^{m}\left(J^{m-\alpha} f(x)\right)
$$


Where

$$
D_{*}^{\alpha} f(x)=J^{m-\alpha}\left(D^{m} f(x)\right),
$$

$$
\boldsymbol{m}-\mathbf{1}<\boldsymbol{\alpha} \leq \boldsymbol{m}, \boldsymbol{m} \in \boldsymbol{N} .
$$

Hence, we have the following properties [5-6]:

$(1) J^{\alpha} J^{\beta} f(x)=J^{\alpha+\beta} f(x)$

$(2) J^{\alpha} J^{\beta} f(x)=J^{\beta} J^{\alpha} f(x)$

(3) $J^{\alpha} t^{\gamma}=\frac{\Gamma(\gamma+1)}{\Gamma(\alpha+\gamma+1)} t^{\alpha+\gamma}, \quad \alpha>0, \gamma>-1, t>0$

The definition of fractional derivative involves an integration which is nonlocal operator (as it is defined on an interval) so fractional derivative is a nonlocal operator. In other word, calculating time-fractional derivative of a function $f(t)$ at some time $t=t_{1}$ requires all the previous history, i.e. all $f(t)$ from $t=0$ to $t=t_{1}$.

\section{Basic idea of homotopy analysis method (HAM)}

The homotopy analysis method (HAM) is proposed first by Liao [10] for solving linear and nonlinear differential and integral equations. Different from perturbation techniques; the (HAM) doesn't depend upon any small or large parameter. This method has been successfully applied to solve many types of nonlinear [11] differential equations, such as projectile motion with the quadratic resistance law. In this paper, (HAM) is applied to solve nonlinear fractional initial-value problem of the non-fatal epidemic model to obtain symbolic approximate solutions for linear and nonlinear differential equations of fractional order. (HAM) is different from all analytical methods; it provides us with a simple way to adjust and control the convergence region [9] of the series solution by introducing the auxiliary parameter $\mathrm{h}$, and the auxiliary function $H(t)$. In fact, it is the auxiliary parameter $h$ that provides us, for the first time, a simple way to ensure the convergence of the series solution. Due to this reason, it seems reasonable to rename $h$ the convergence-control parameter [3]. It should be emphasized that, without the use of the convergence-parameter $h$, one had to assume that the homotopy series is convergent. However, with the use of the convergence-parameter $h$, such an assumption is unnecessary; because it seems that one can always choose a proper value of $h$ to obtain convergent homotopyseries solution. So, the use of the convergence-parameter $h$ in the zeroth-order deformation equation greatly modifies the early homotopy analysis method. Since then, the homotopy analysis method has been developing greatly, and more generalized zeroth-order deformation equations are suggested by Liao [9-10]. 
The Adomain decomposition method (ADM), the homotopy perturbation method (HPM), the variational iteration method (VIM) and other methods have been used to provide analytical approximation to linear and nonlinear problems. However, the convergence region of the corresponding results is rather small as shown in [9]. The zeroth-order deformation equations and is rather general. Using them, Liao proved that the (HAM) logically contains other previous nonperturbation methods, such as Lyapunov's artificial small parameter method, the d-expansion method and Adomian's decomposition method. Thus, the (HAM) unifies the previous non-perturbation methods. Besides, the so-called "homotopy perturbation method" (proposed in 1998) is exactly the same as the early homotopy analysis method (proposed in 1992) and is a special case of the late homotopy analysis method in case of $h=-1$.

Consider the following system of (FDE):

$D^{\alpha_{i}}\left(u_{i}(t)\right)=f_{i}\left(t, u_{1}, \ldots, u_{n}\right), \quad i=1,2,3, \ldots, n, \quad 0 \leq \alpha_{i} \leq 1$

Subject to the initial conditions:

$$
u_{i}(0)=a_{i}, \quad i=1,2, \ldots, n
$$

Liao [10] constructed the so-called zeroth-order deformation equation:

$$
(1-q) \mathcal{L}_{i}\left[\phi_{i}(t, q)-u_{i 0}(t)\right]=q h_{i} H_{i}(t) N_{i}\left[\phi_{i}(t, q)\right], \quad i=1,2,3, \ldots n
$$

subject to the initial conditions:

$$
\phi_{i}(0, q)=a_{i}
$$

where $q \in[0,1]$ is an embedding parameter, $\mathrm{N}_{\mathrm{i}}$ are nonlinear operators, $\mathcal{L}_{\mathrm{i}}$ are auxiliary linear operators satisfy $\mathcal{L}_{\mathrm{i}}(0)=0, u_{i 0}(t)$ are initial guesses satisfy the initial conditions (3), $h_{i} \neq 0$ are auxiliary parameters, $H_{i}(t) \neq 0$ are auxiliary functions, $\phi_{i}(t, q)$ are unknown functions. It should be emphasized that one has great freedom to choose, the auxiliary linear operators $\mathcal{L}_{\mathrm{i}}$, the auxiliary parameters $\mathrm{h}_{\mathrm{i}}$ and the auxiliary functions $H_{i}$. Obviously, when $q \neq 0$, since $u_{i 0}(t)$ satisfy the initial conditions $(3)$ and $\mathcal{L}_{\mathrm{i}}(0)=0$, we have

$$
\phi_{i}(t, 0)=u_{i 0}(t), \quad i=1,2,3, \ldots, n \text {, }
$$

when $q=1$, since $h_{i} \neq 0$ and $H_{i}(t) \neq 0$, the zeroth-order deformation equation (4) and (5) are equivalent to (2) and (3), hence

$$
\phi_{i}(t, 1)=u_{i 0}(t), \quad i=1,2,3, \ldots, n \text {. }
$$


Thus, as $q$ increasing from 0 to 1 , the solutions $\phi_{i}(t, q)$ various from $u_{i 0}(t)$ to $u_{i}(t)$. Expanding $\phi_{i}(t, q)$ in Taylor series with respect to the embedding parameter $q$, one has

$$
\phi_{i}(t, q)=u_{i 0}(t)+\sum_{m=1}^{\infty} u_{i m}(t) q^{m}, \quad i=1,2,3, \ldots, n,
$$

Where

$$
u_{i m}(t)=\left.\frac{1}{m !} \frac{\partial^{m} \phi_{i}(t, q)}{\partial q^{m}}\right|_{q=0}, i=1,2,3, \ldots, n
$$

Assume that the auxiliary parametersh $h_{i}$, the auxiliary functions $H_{i}(t)$, the initial approximations $u_{i 0}(t)$ and the auxiliary linear operators $\mathcal{L}_{\mathrm{i}}$ are properly chosen so that the series (8) converges at $q=1$. Then at $q=1$, and by (7) the series (8) becomes

$$
u_{i}(t)=u_{i 0}(t)+\sum_{m=1}^{\infty} u_{i m}(t), \quad i=1,2,3, \ldots, n
$$

and now define the vector

$$
\vec{u}_{i}=\left\{u_{i 0}, u_{i 1}, u_{i 2}, \ldots, u_{i j}\right\}, \quad i=1,2,3, \ldots j
$$

Differentiating equations (4) $\mathrm{m}$ times with respect to the embedding parameter $q$, then setting $q=0$ and dividing them by $m$ !, finally using (9), we have the socalled $m$ th -order deformation equations:

$$
\mathcal{L}_{\mathrm{i}}\left[\mathrm{u}_{\mathrm{im}}-\mathcal{X}_{\mathrm{m}} \mathrm{u}_{\mathrm{i}(\mathrm{m}-1)}(\mathrm{t})\right]=\mathrm{h}_{\mathrm{i}} \mathrm{H}_{\mathrm{i}}(\mathrm{t}) \Re_{\mathrm{im}}\left(\overrightarrow{\mathrm{u}}_{\mathrm{i}(\mathrm{m}-1)}(\mathrm{t})\right), \quad \mathrm{i}=1,2, \ldots, \mathrm{n},
$$

Subject to the conditions:

$$
u_{i m}(0)=0, \quad i=1,2, \ldots, n, m=1,2,3, \ldots
$$

Where

$$
\mathfrak{R}_{i m}\left(\vec{u}_{i(m-1)}(t)\right)=\left.\frac{1}{(m-1) !} \frac{\partial^{m-1} N_{i}\left(\phi_{i}(t, q)\right)}{\partial q^{m-1}}\right|_{q=0}
$$

and

$\chi_{m}= \begin{cases}0, & m \leq 1 \\ 1, & m>1\end{cases}$ 
If we choose the linear operator $\mathcal{L}_{i}=D^{\alpha_{i}}$ then according to (12), we have

$$
J^{\alpha_{i}} D^{\alpha_{i}}\left[u_{i m}-x_{m} u_{i(m-1)}(t)\right]=h_{i} J^{\alpha_{i}}\left[H_{i}(t) \Re_{i m}\left(\vec{u}_{i(m-1)}(t)\right)\right]
$$

Finally it seems that, as long as a nonlinear fractional order differential equation has at least one solution, then one can always construct a kind of zeroth-order deformation equation to get convergent homotopy-series solution as

$$
u_{i m}=\chi_{m} u_{i(m-1)}(t)+h_{i} J^{\alpha_{i}}\left[H_{i}(t) \Re_{i m}\left(\vec{u}_{i(m-1)}(t)\right)\right]
$$

and

$$
u_{i}=u_{i 0}+u_{i 1}+u_{i 2}+u_{i 3+\cdots}
$$

\section{$4 \quad$ Fractional order non-fatal epidemic model}

Now we introduce fractional-order into the model of epidemic model by replacing the first time derivative term by a fractional derivative of order $\alpha_{1}, \alpha_{1}, \alpha_{3}>0$. The new system is described by the following system of FODE:

$$
\begin{aligned}
& D^{\alpha_{1}}(x)=-\beta x y, \\
& D^{\alpha_{2}}(y)=\beta x y-\gamma y, \\
& D^{\alpha_{3}}(z)=\gamma y .
\end{aligned}
$$

Subject to the initial values

$$
x(0)=N_{1}, y(0)=N_{2}, z(0)=N_{3} .
$$

This paper attempts to find numerical solution for a general class of fractional order model of non-fatal epidemic model. For this purpose the paper summarizes specific techniques for HAM, as well as the applications of Caputo fractional calculus.

The reason of using fractional order differential equations (FOD) is that FOD are naturally related to systems with memory which exists in most biological systems. Also they are closely related to fractals which are abundant in biological systems. The results derived of the fractional system (18) are of a more general nature. We would like to put your attention that time fractional derivatives change also the solutions we usually get in standard system (1). The concept of fractional or non-integer order derivation and integration can be traced back to the genesis of integer order calculus itself $[3,5]$. Most of the mathematical theory applicable to the study of non-integer order calculus was developed through the end of 19th century. However it is in the past hundred years that the most intriguing leaps in engineering and scientific application have been found. The calculation technique 
has in some cases had to change to meet the requirement of physical reality. The derivatives are understood in the Caputo sense. The general response expression contains a parameter describing the order of the fractional derivative that can be varied to obtain various responses. In view of the homotopy analysis method presented above, if we select the auxiliary functions $H_{1}=H_{2}=H_{3}=1$, we can construct the homotopy:

$$
\begin{aligned}
& \mathfrak{R}_{1 m}\left(\vec{x}_{(m-1)}(t)\right)=D^{\alpha_{1}} x_{m-1}+\beta \sum_{i=0}^{m-1} x_{i} y_{m-1-i}, \\
& \mathfrak{R}_{2 m}\left(\vec{y}_{(m-1)}(t)\right)=D^{\alpha_{2}} y_{m-1}-\beta \sum_{i=0}^{m-1} x_{i} y_{m-1-i}+\gamma y_{m-1}, \\
& \mathfrak{R}_{3 m}\left(\vec{z}_{(m-1)}(t)\right)=D^{\alpha_{3}} z_{m-1}-\gamma y_{m-1},
\end{aligned}
$$

Consequently, we have

$$
\begin{gathered}
x_{m}=x_{m} x_{m-1}+h_{1} J^{\alpha_{1}}\left[\Re_{1 m}\left(\vec{x}_{(m-1)}(t)\right)\right], \\
y_{m}=x_{m} y_{m-1}+h_{2} J^{\alpha_{2}}\left[\Re_{2 m}\left(\vec{y}_{(m-1)}(t)\right)\right], \\
z_{m}=x_{m} z_{m-1}+h_{3} J^{\alpha_{3}}\left[\Re_{3 m}\left(\vec{z}_{(m-1)}(t)\right)\right] .
\end{gathered}
$$

\section{$5 \quad$ Numerical results and discussion}

For comparison with the results done by Biazar [1], the following values, for parameters, are considered.

Table 1: Parameters values

\begin{tabular}{lc}
\hline Parameters & Values \\
\hline Initial population of $x(t)$, who are susceptible & $N_{1}=20$ \\
Initial population of $y(t)$, who are infective & $N_{2}=15$ \\
Initial population of $z(t)$, who are immune & $N_{3}=10$ \\
Rate of change of susceptible population to infective population & $\beta=0.01$ \\
Rate of change of infective population to immune population & $\gamma=0.02$ \\
\hline
\end{tabular}

The first few components of the homotopy analysis solution $x(t), y(t)$ and $z(t)$, are calculated. We computed the first three terms of the HAM series solution for the system (18). We present two of them as follows: 


$$
\begin{aligned}
& x_{1}=\frac{3 t^{\alpha_{1}} h_{1}}{\Gamma\left(1+\alpha_{1}\right)}, y_{1}=\frac{-2.7 t^{\alpha_{2}} h_{2}}{\Gamma\left(1+\alpha_{2}\right)}, z_{1}=\frac{-0.3 t^{\alpha_{3}} h_{3}}{\Gamma\left(1+\alpha_{3}\right)} \\
& x_{2}=\frac{3 t^{\alpha_{1}} h_{1}}{\Gamma\left(1+\alpha_{1}\right)}+\frac{3 t^{\alpha_{1}} h_{1}^{2}}{\Gamma\left(1+\alpha_{1}\right)}+\frac{0.45 t^{2 \alpha_{1}} h_{1}^{2}}{\Gamma\left(1+2 \alpha_{1}\right)}-\frac{0.54 t^{\alpha_{1}+\alpha_{2}} h_{1} h_{2}}{\Gamma\left(1+\alpha_{1}+\alpha_{2}\right)} \\
& y_{2}=\frac{-2.7 t^{\alpha_{2}} h_{2}}{\Gamma\left(1+\alpha_{2}\right)}-\frac{0.45 t^{\alpha_{1}+\alpha_{2}} h_{1} h_{2}}{\Gamma\left(1+\alpha_{1}+\alpha_{2}\right)}-\frac{2.7 t^{\alpha_{2}} h_{2}^{2}}{\Gamma\left(1+\alpha_{2}\right)}+\frac{0.486 t^{2 \alpha_{2}} h_{2}^{2}}{\Gamma\left(1+2 \alpha_{2}\right)} \\
& z_{2}=\frac{-0.3 t^{\alpha_{3}} h_{3}}{\Gamma\left(1+\alpha_{3}\right)}+\frac{0.045 t^{\alpha_{2}+\alpha_{3}} h_{1} h_{2}}{\Gamma\left(1+\alpha_{2}+\alpha_{3}\right)}-\frac{0.3 t^{\alpha_{2}} h_{3}^{2}}{\Gamma\left(1+\alpha_{3}\right)}
\end{aligned}
$$

Thus, the HAM series solution of the given system (18) can be given by:

$x=x_{0}+x_{1}+x_{2}+x_{3}+\cdots$

$y=y_{0}+y_{1}+y_{2}+y_{3}+\cdots$

$z=z_{0}+z_{1}+z_{2}+z_{3}+\cdots$

One can choose the values of $h_{1}, h_{2}, h_{3}$ from the so called h-curves. Fig.1. represents some of the h-curves while Fig.2. represents the HAM 3rd order series solution of system (18) in case of $h_{1}=h_{2}=h_{3}=h$ and $\alpha_{1}=\alpha_{2}=\alpha_{3}=\alpha$. At $h=-1, \alpha=1$ we obtain three terms approximations which are the same solutions obtained in [1] using (ADM), in [7] using (VIM), and in [8] using (HPM) as follows

$$
\begin{aligned}
& x=20-3 t-0.045 t^{2}+0.02805 t^{3} \\
& y=15+2.7 t+0.018 t^{2}-0.02817 t^{3} \\
& z=10+0.3 t+0.027 t^{2}+0.00012 t^{3}
\end{aligned}
$$
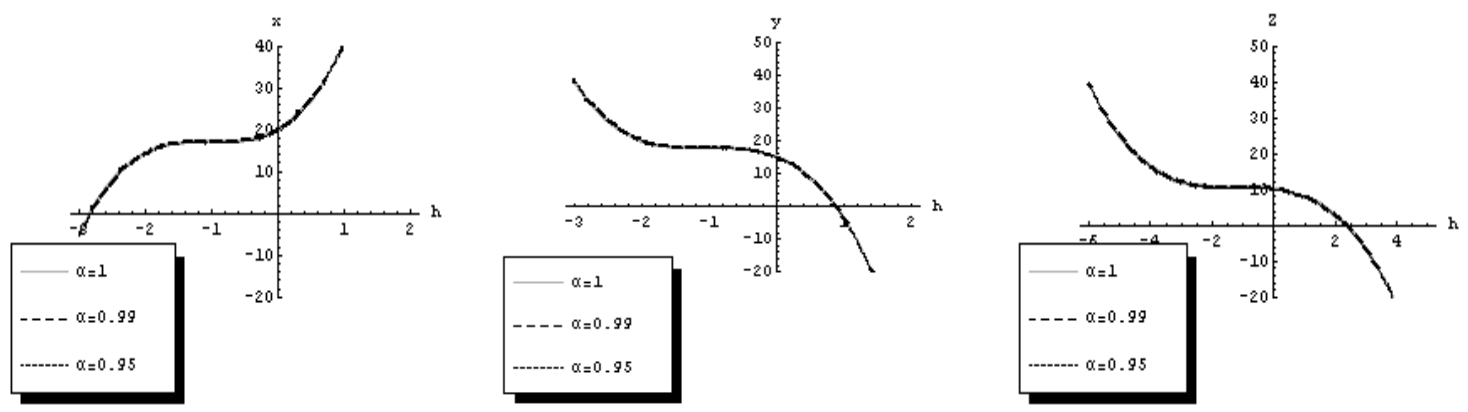

Fig. 1: The $h$-curves of the system (18) at different values of $\alpha$.
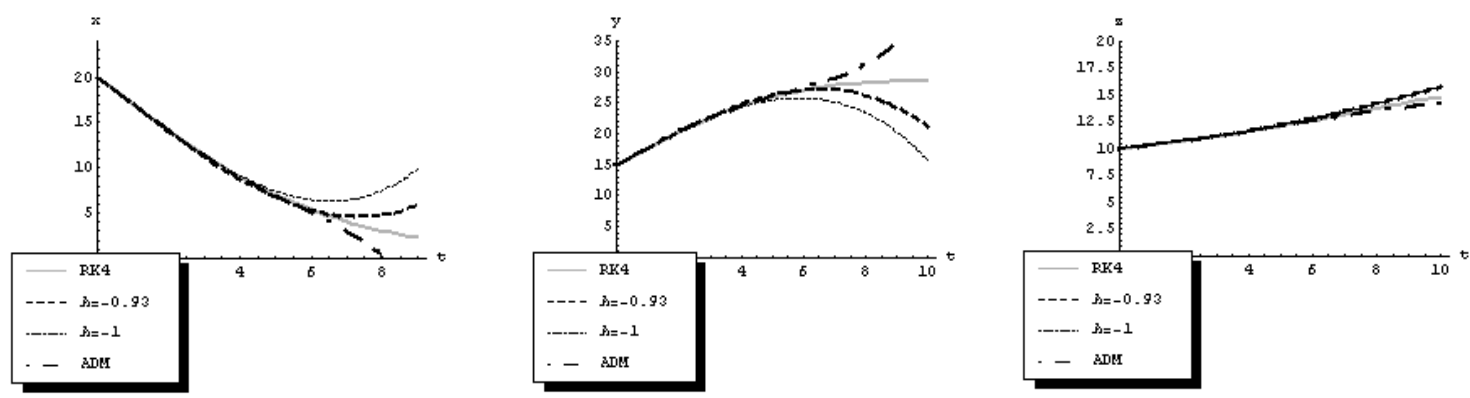

Fig. 2: The numerical results at $\alpha=1$. 

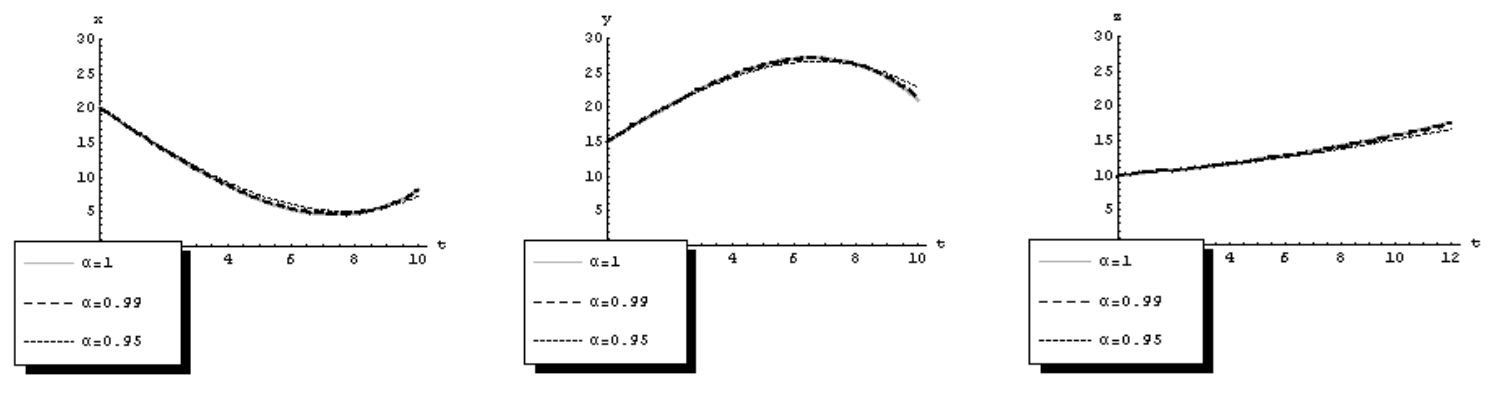

Fig.3 : The numerical results at different values of $\alpha$ and $h=-0.93$

Table 2: The number of susceptible individuals $x(t)$ in case of $h_{i}=-1, \beta=$ $0.01, \gamma=0.02$.

\begin{tabular}{ccccccc}
\hline$t$ & $\alpha_{1}=1$ & $\alpha_{1}=0.99$ & $\alpha_{1}=0.95$ & $\alpha_{1}=1$ & $\alpha_{1}=1$ & $\alpha_{1}=0.99$ \\
& $\alpha_{2}=1$ & $\alpha_{2}=0.99$ & $\alpha_{2}=0.95$ & $\alpha_{2}=0.99$ & $\alpha_{2}=0.95$ & $\alpha_{2}=0.99$ \\
& $\alpha_{3}=1$ & $\alpha_{3}=0.99$ & $\alpha_{3}=0.95$ & $\alpha_{3}=0.99$ & $\alpha_{3}=0.95$ & $\alpha_{3}=0.95$ \\
\hline & & & & & & \\
0 & 20.0000 & 20.0000 & 20.0000 & 20.0000 & 20.0000 & 20.0000 \\
1 & 16.9831 & 16.9704 & 16.9213 & 16.9807 & 16.9712 & 16.9704 \\
2 & 14.0444 & 14.0616 & 14.1336 & 14.0419 & 14.0321 & 14.0616 \\
3 & 11.3524 & 11.4109 & 11.6459 & 11.3540 & 11.3615 & 11.4109 \\
4 & 9.07520 & 9.17302 & 9.56071 & 9.08472 & 9.12393 & 9.17302 \\
5 & 7.38125 & 7.50352 & 7.98741 & 7.40056 & 7.47862 & 7.50352 \\
6 & 6.43880 & 6.55779 & 7.03558 & 6.46736 & 6.58109 & 6.55779 \\
7 & 6.41615 & 6.49071 & 6.81354 & 6.45041 & 6.58445 & 6.49071 \\
8 & 7.48160 & 7.45661 & 7.42799 & 7.51463 & 7.63989 & 7.45661 \\
9 & 9.80345 & 9.60930 & 8.98403 & 9.82465 & 9.89711 & 9.60930 \\
10 & 13.5500 & 13.1021 & 11.5852 & 13.5448 & 13.5045 & 13.1021 \\
11 & 18.8896 & 18.0879 & 15.3336 & 18.8393 & 18.6094 & 18.0879 \\
12 & 25.9904 & 24.7191 & 20.3300 & 25.8719 & 25.3582 & 24.7191 \\
\hline
\end{tabular}


Table 3: The number of infected individuals $y(t)$ in case of $h_{i}=-1, \beta=$ $0.01, \gamma=0.02$.

\begin{tabular}{ccccccc}
\hline$t$ & $\alpha_{1}=1$ & $\alpha_{1}=0.99$ & $\alpha_{1}=0.95$ & $\alpha_{1}=1$ & $\alpha_{1}=1$ & $\alpha_{1}=0.99$ \\
& $\alpha_{2}=1$ & $\alpha_{2}=0.99$ & $\alpha_{2}=0.95$ & $\alpha_{2}=0.99$ & $\alpha_{2}=0.95$ & $\alpha_{2}=0.99$ \\
& $\alpha_{3}=1$ & $\alpha_{3}=0.99$ & $\alpha_{3}=0.95$ & $\alpha_{3}=0.99$ & $\alpha_{3}=0.95$ & $\alpha_{3}=0.95$ \\
\hline & & & & & & \\
0 & 15.0000 & 15.0000 & 15.0000 & 15.0000 & 15.0000 & 15.0000 \\
1 & 17.6898 & 17.7007 & 17.7428 & 17.7031 & 17.7550 & 17.7007 \\
2 & 20.2466 & 20.2306 & 20.1636 & 20.2329 & 20.1742 & 20.2306 \\
3 & 22.5014 & 22.4498 & 22.2431 & 22.4438 & 22.2101 & 22.4498 \\
4 & 24.2851 & 24.2029 & 23.8775 & 24.1748 & 23.7357 & 24.2029 \\
5 & 25.4287 & 25.3336 & 24.9570 & 25.2646 & 24.6190 & 25.3336 \\
6 & 25.7633 & 25.6861 & 25.3717 & 25.5515 & 24.7279 & 25.6861 \\
7 & 25.1197 & 25.1047 & 25.0131 & 24.8746 & 23.9319 & 25.1047 \\
8 & 23.3290 & 23.4346 & 23.7743 & 23.0731 & 22.1018 & 23.4346 \\
9 & 20.2221 & 20.5214 & 21.5500 & 19.9868 & 19.1100 & 20.5214 \\
10 & 15.6300 & 16.2113 & 18.2366 & 15.4558 & 14.8304 & 16.2113 \\
11 & 9.38373 & 10.3506 & 13.7317 & 9.32030 & 9.13801 & 10.3506 \\
12 & 1.31424 & 2.78651 & 7.93438 & 1.42114 & 1.90918 & 2.78651 \\
\hline
\end{tabular}

Table 4: The number of isolated individuals $z(t)$ in case of $h_{i}=-1, \beta=$ $0.01, \gamma=0.02$.

\begin{tabular}{ccccccc}
\hline$t$ & $\alpha_{1}=1$ & $\alpha_{1}=0.99$ & $\alpha_{1}=0.95$ & $\alpha_{1}=1$ & $\alpha_{1}=1$ & $\alpha_{1}=0.99$ \\
& $\alpha_{2}=1$ & $\alpha_{2}=0.99$ & $\alpha_{2}=0.95$ & $\alpha_{2}=0.99$ & $\alpha_{2}=0.95$ & $\alpha_{2}=0.99$ \\
& $\alpha_{3}=1$ & $\alpha_{3}=0.99$ & $\alpha_{3}=0.95$ & $\alpha_{3}=0.99$ & $\alpha_{3}=0.95$ & $\alpha_{3}=0.95$ \\
\hline & & & & & & \\
0 & 10.0000 & 10.0000 & 10.0000 & 10.0000 & 10.0000 & 10.0000 \\
1 & 10.3271 & 10.3289 & 10.3359 & 10.3289 & 10.3360 & 10.3348 \\
2 & 10.7090 & 10.7078 & 10.7028 & 10.7079 & 10.7031 & 10.7019 \\
3 & 11.1462 & 11.1393 & 11.1110 & 11.1394 & 11.1112 & 11.1129 \\
4 & 11.6397 & 11.6241 & 11.5617 & 11.6240 & 11.5610 & 11.5701 \\
5 & 12.1900 & 12.1628 & 12.0556 & 12.1622 & 12.0521 & 12.0744 \\
6 & 12.7979 & 12.7561 & 12.5927 & 12.7544 & 12.5841 & 12.6265 \\
7 & 13.4642 & 13.4046 & 13.1734 & 13.4011 & 13.1563 & 13.8764 \\
8 & 14.1894 & 14.1088 & 13.7977 & 14.1025 & 13.7680 & 14.5752 \\
9 & 14.9745 & 14.8693 & 14.4659 & 14.8591 & 14.4185 & 15.3238 \\
10 & 15.8200 & 15.6866 & 15.1782 & 15.6712 & 15.1071 & 15.3238 \\
11 & 16.7267 & 16.5615 & 15.9347 & 16.5392 & 15.8330 & 16.1227 \\
12 & 17.6954 & 17.4943 & 16.7356 & 17.4633 & 16.5953 & 16.9724 \\
\hline
\end{tabular}




\section{Conclusion}

The results plotted in Fig.1, Fig. 2, Fig. 3, tables 2,3,4 show that while the number of susceptible population increases the population of who are infective decreases in the period of the epidemic, meanwhile the number of immune population increases. But the size of the population over the period of the epidemic is constant. Fractional order results (see tables 2-3) show the realistic biphasic decline behavior of the non-fatal epidemic but at a slower rate. As a definition of fractional calculus: $\lim _{\alpha \rightarrow 1} D^{\alpha} f(t)=D f(t)$ has been provided. In the presented problem, The number of susceptible individuals $x(t)$, the number of infected individuals $y(t)$, the number of isolated individuals $z(t)$ have been obtained, therefore when $\alpha \rightarrow 1$ the solution of the fractional model (18) $x_{\alpha}(t)$, $y_{\alpha}(t)$ and $z_{\alpha}(t)$, reduce to the standard solution $x(t), y(t)$ and $z(t)$.

We demonstrated the accuracy and efficiency of (HAM) by solving the presented problem in case of integer order or fractional order. (HAM) is accurate, fast and reliable for such problems. There are some important points to make here. First, we have great freedom to choose the auxiliary linear operators $\mathcal{L}_{\mathrm{i}}$, the auxiliary parameters $h_{i}$ and the auxiliary functions $H_{i}$. Second, (HAM) was shown to be a simple, yet powerful analytic numeric scheme for handling systems of fractional order. The results obtained by (HAM) are the same results obtained in [1] using (ADM), in [7] using (VIM), and in [8] using (HPM) at $h=-1$. In other words (ADM), (VIM), (HPM) are special cases of (HAM). A comparison in fig. 2 was presented between the HAM $3^{\text {rd }}$ order results, ADM $5^{\text {th }}$ order results obtained in [1], and RK4 method. At $h=-0.93$. HAM 3rd order results has excellent agreement with the RK4 results and more convergent than order ADM 5 th order results.

\section{References}

[1] J. Biazar, Solution of the epidemic model by Adomian decomposition method, Applied Mathematics and Computation, 173 (2) (2006) 1101-1106.

[2] A.A.M. Arafa, S.Z. Rida and M. Khalil, Fractional modeling dynamics of HIV and CD4+ T-cells during primary infection, Nonlinear Biomedical Physics, 6 ( 2012) 1-7.

[3] Hany N. Hassan, and Magdy A. El-Tawil, A new technique of using homotopy analysis method for solving high-order nonlinear differential equations, Math. Meth. Appl. Sci. 34 (2011) 728-742.

[4] D.W. Jordan, P. Smith, Nonlinear Ordinary Differential Equations, third ed., Oxford University Press, 1999.

[5] Mohammad Zurigat, Shaher Momani, Ahmad Alawneh, Analytical approximate solutions of systems of fractional algebraic differential 
equations by homotopy analysis method, Computers and Mathematics with Applications 59(2010) 1227-1235.

[6] Mohammad Zurigat, Shaher Momani , Zaid Odibat, Ahmad Alawneh, The homotopy analysis method for handling systems of fractional differential equations, Applied Mathematical Modelling 34 (2010) 24-35.

[7] M. Rafei, H. Daniali, D.D. Ganji, Variational iteration method for solving the epidemic model and the prey and predator problem, Applied Mathematics and Computation 186 (2007) 1701-1709.

[8] M. Rafei , D.D. Ganji, H. Daniali, Solution of the epidemic model by homotopy perturbation method, Applied Mathematics and Computation, 187 (2007) 1056-1062.

[9] S.J. Liao, Notes on the homotopy analysis method: Some definitions and theorems, Commun Nonlinear Sci Numer Simulat 14 (2009) 983-997.

[10] S.J. Liao, The Proposed Homotopy Analysis Technique for the Solution of Nonlinear Problems, Ph.D. Thesis, Shanghai Jiao Tong University, 1992.

[11] Zaid Odibat, Shaher Momani , Hang Xu, A reliable algorithm of homotopy analysis method for solving nonlinear fractional differential equations, Applied Mathematical Modelling 34 (2010) 593-600. 